\title{
Pharmacogenomics in the psychiatric population
}

\begin{abstract}
Recent research has revealed that patients may metabolize the same drug differently. Why and how does this occur? Investigation into the expanding field of pharmacogenomics can help answer these two questions. Pharmacogenomics is the study of all genes in the genome that may determine drug response. ${ }^{1}$ Looking into studies where psychiatric patients have been diagnosed with the same disease, it has been observed that a large sample of patients react differently to similar or the same medications. In pharmacogenomics of the psychiatric population, drugs are divided into three categories based on safety and effectiveness: red category, yellow category, and green category; red-zone, yellow-zone, and green-zone. The subjects of the studies cited herein are a sampling of those who took either red, yellow, or green categorized FDA-approved drugs. There is also a small sample of subjects who took a combination of yellow and green drugs simultaneously. However, there are no subjects that took a combination of red and yellow or a combination of red and green; and there are also no subjects that took drugs from all three categories. Drugs that treat depression are the focus of this research, as psychiatric medicines, such as antidepressants, have a large sampling of patients who have or have not done well with the various medications. Pharmacogenomic testing will help treat patients more effectively using medications that better correspond to the patient's genetic makeup. This results in a more personalized approach to treatment with medicines.
\end{abstract}

Keywords: gene, personalized medicine, pharmacogenomics, psychiatric
Volume 9 Issue 3 - 2018

\author{
Olubunmi A Omoshebi,' Nicholas A Kerna, ${ }^{1,2}$ \\ Tony L Brown ${ }^{3}$ \\ 'College of Medicine, University of Science, Art and Technology, \\ USA \\ ${ }^{2}$ Department of Psychiatry, Suriwongse Medical Center, Thailand \\ ${ }^{3}$ Harvard University, USA
}

Correspondence: Nicholas A Kerna, College of Medicine, University of Science, Art and Technology, 7583 Sourdough Dr. Morrison, CO 80465, USA, Email nicholas.kerna@usat.edu

Received: May 29, 2017 | Published: May 04, 2018
Abbreviations: ADHD, attention deficit hyperactivity disorder; CPGx, combination pharmacogenomics; DNA, deoxyribonucleic acid; JRA, juvenile rheumatoid arthritis; MTHFR, methylenetetrahydrofolate reductase; OA, osteoarthritis; OCD, obsessive-compulsive disorder; PMDD, premenstrual dysmorphic disorder; PTSD, post-traumatic stress disorder; RA, rheumatoid arthritis; SNRI, serotonin and norepinephrine reuptake inhibitors; SSRI, selective serotonin reuptake inhibitor; URM, ultra-rapid metabolizer

\section{Introduction}

Individual patients can respond differently to the same or similar medication. The differences can be due to a multitude of factors: age, ethnicity, body mass, nutrition, metabolizing enzymes, and/or genetics. Studying the effects of genetic factors on medications might help physicians select the best medication for a particular patient. The study of human genes and how they affect medication and efficacy is termed, pharmacogenomics. One of the most important features of pharmacogenomics is that it can be used to predict, and thus prevent, adverse drug reactions that can seriously affect a patient's quality of life. ${ }^{2}$ Pharmacogenomics can also be used to look into toxicity levels in patients, thus reducing overdosing on a prescribed medication. Pharmacogenomics is a sector of what is called, "personalized medicine" which could change the paradigm of medical care for individual patients for the better. The more specific patient treatment can become, the more likelihood for improved outcomes. The Golden Helix Institute of Biomedical Research has deployed several genomic databases as a web service so that healthcare providers and researchers can access the many studies and results. ${ }^{3}$ This type of intercommunication and sharing of information is essential to the field of pharmacogenomics as it moves forward. The summary herein is focused on psychiatric patients and the medications that are typically used.

\section{Summary}

Many metabolizing genes influence drug response. Cytochrome P450 2D6 (CYP2D6) was the first gene that identified the increase and decrease of metabolic capacity in a psychiatric patient. ${ }^{2}$ CYP2D6 is located on chromosome 22, and this gene is highly polymorphic. The polymorphism of CYP2D6 can change the way a patient metabolizes a particular drug. Patients are classified as poor metabolizers, intermediate metabolizers, or ultra-rapid metabolizers. Patients with multiple gene copies of CYP2D6 will metabolize a particular drug more rapidly (these are the ultra-rapid metabolizers), and will also need a slightly lower dosage of the medication than individuals without the gene or with less alleles. Poor metabolizers will metabolize a particular drug slowly, and are more susceptible to having more side effect/adverse drug effects. This is extremely important in psychiatry because $50 \%$ of the drugs prescribed are drugs that are primarily metabolized by CYP2D6, such as the SSRIs, SNRIs, and TCAs.

Most antidepressant medications are metabolized by two or more CYP enzymes. The phenotypes are used by the GeneSight test to predict how each patient is likely to respond to each panel of medication. The combination approach can compensate for the variability and relatively small effect sizes for associations between single alleles and psychiatric medication response by aggregating the more consistently predictive genes, such as CYP2C19, CYP2D6, and CYP1A2. Just over seven percent $(7.3 \%)$ of Caucasians are poor metabolizers or ultra-rapid metabolizers for CYP2D6, 3.7\% are poor metabolizers for CYP2C19, and 49\% are ultra-rapid metabolizers for CYP1A2. ${ }^{5}$ 


\section{Conclusion}

The concept that some patients will react differently than other patients to certain medications is not a novel concept. Hippocrates was one of the first advocates of personalized medicine; the theory of the four humors is a classic historical example of this ideology. ${ }^{4,6}$ Technology has advanced to a point where we can prove what Hippocrates could only hypothesize, and can build a more advanced, patient-specific form of care. It is essential to a patient's overall health and quality of life that the attitude of one size fits allparticularly in the area of psychiatry-must be removed entirely from the healthcare field. ${ }^{6}$ In psychiatry, with personalized medicine and the use of pharmacogenomics, more effective drug treatments can be developed. Safety precautions of the patient pool will be known. Drugs can be more specific. ${ }^{7}$ The key will be to develop greater precision in the use of pharmacogenomics for the practitioner. ${ }^{8}$ The strength of studies and research directly affect how well a concept, like pharmacogenomics, is received by healthcare professionals. The clinical studies, reported herein, have shown the benefit of finding genes that affect the way a psychiatric patient metabolizes their medication. Performing additional research, as well as assisting in the collation of information into the public repositories, will help patients be better tested for their ability to metabolize medications. Knowing this information will allow for the building of profiles; so that, in addition to the effects of the single alleles, combinations can also be considered, as they too may be indicators for certain conditions and, subsequently, a particular drug prescription. Performing pharmacogenomic testing on psychiatric patients will help the physician determine a management plan that would best help relieve the patients symptoms while reducing or eliminating undesirable side effects. This will ultimately improve the lives of patients, and reduce the cost of medical care which occurs through the trial-anderror method that is commonly used today in the management of psychiatric patients.

\section{Acknowledgments}

None.

\section{Conflict of interest}

The authors declare the research was conducted in the absence of any commercial of financial relationships that could be construed as a potential conflict of interest.

\section{References}

1. Evans WE, Relling MV. Pharmacogenomics: translating functional genomics into rational therapeutics. Science. 1999;286(5439):487-491.

2. Squassina A, Severino G, Grech G, et al. Golden Helix Pharmacogenomics Days: educational activities on pharmacogenomics and personalized medicine. Pharmacogenomics. 2012;13(5):525-528.

3. Mitropoulos K, Innocenti F, van Schaik RH, et al. Golden Helix Institute of Biomedical Research: Interdisciplinary research and educational activities in pharmacogenomics and personalized medicine. Pharmacogenomics. 2012;13(4):387-392.

4. Abul-Husn NS, Owusu Obeng A, Sanderson SC, et al. Implementation and utilization of genetic testing in personalized medicine. Pharmacogenomics Pers Med. 2014;7:227-240.

5. Mrazek DA. Psychiatric pharmacogenomic testing in clinical practice. Dialogues Clin Neurosci. 2010;12(1):69-76.

6. Gupta PD. Pharmacogenetics, Pharmacogenomics and Ayurgenomics for Personalized Medicine: A Paradigm Shift. Indian J Pharm Sci. 2015;77(2):135-141.

7. Mirsadeghi S, Larijani B. Personalized Medicine: Pharmacogenomics and Drug Development. Acta Med Iran. 2017;55(3):150-165.

8. $\mathrm{Xu} \mathrm{R}$, Wang Q. A semi-supervised approach to extract pharmacogenomics-specific drug-gene pairs from biomedical literature for personalized medicine. J Biomed Inform. 2013;46(4):585-593. 